\title{
OSCILLATIONS IN ONE-DIMENSIONAL ELASTICITY WITH SURFACE ENERGY
}

\author{
BY \\ IRENE FONSECA, JACK SCHAEFFER, AND MIKHAIL M. SHVARTSMAN \\ Department of Mathematics, Carnegie Mellon University, Pittsburgh, Pennsylvania
}

\begin{abstract}
The characterization of the oscillatory behavior of solutions of a semilinear equation in one space dimension is obtained. In this work the model equation for a material undergoing a phase transition encompasses a surface energy term and first-order memory effects.
\end{abstract}

1. Introduction. In recent years there has been a remarkable progress in the understanding of nonconvex variational problems, motivated in part by the study of phase transitions in solids. Consider a one-dimensional elastic bar in a hard device with nonconvex underlying elastic energy density

$$
W(p):=\frac{1}{4}\left(p^{2}-1\right)^{2},
$$

where $p:=u_{x}(x, t)$ is the strain and $u(x, t)$ stands for the longitudinal displacement of a particle with reference coordinate $x$ at time $t$. Assume further that the reference configuration occupies the interval $(0,1)$.

Clearly, any quasi-static distribution of strains with values \pm 1 provides zero minimal total energy

$$
\mathcal{I}_{0}(u):=\int_{0}^{1} W\left(u_{x}(x)\right) d x .
$$

As is well known, in the sharp interface setting this nonuniqueness may be resolved by penalizing the formation of interfaces (i.e., jumps in $u_{x}$ ), while in the case where transitions occur across smooth layers one may consider singular perturbations

$$
\mathcal{I}_{1}(u):=\int_{0}^{1}\left[W\left(u_{x}(x)\right)+\frac{\gamma}{2}\left(u_{x x}(x)\right)^{2}\right] d x
$$

for small $\gamma>0$. As pointed out by Truskinovsky and Zanzotto [TZ], this model admits a unique minimizer with exactly one interface. This is not in agreement with experiments where multiple interfaces are present and subject to mechanisms that prevent

Received January 15, 1997.

1991 Mathematics Subject Classification. Primary 35B05, 35B30, 35Q72.

Key words and phrases. Oscillations, div-curl lemma. 
variants from becoming finer and finer. In light of this remark, and motivated by models previously introduced in $[\mathrm{Pe}]$ and $[\mathrm{BHJPS}]$, we consider the evolution problem

$$
u_{t t}=\left[\sigma\left(u_{x}\right)+\beta u_{x t}-\gamma u_{x x x}\right]_{x}-\alpha u
$$

where

$$
\sigma(p):=W^{\prime}(p)=p^{3}-p
$$

is the stress, $\beta>0, \gamma>0, \alpha \geq 0$ corresponds to the stiffness of the elastic matrix, and where $u_{x x t}, u$, and $u_{x x x x}$ account for, respectively, viscoelastic damping, forcing term, and local surface energy penalization. The associated potential energy is given by

$$
\mathcal{I}(u)(t):=\frac{1}{4} \int_{0}^{1}\left(u_{x}^{2}(x, t)-1\right)^{2} d x+\frac{\alpha}{2} \int_{0}^{1} u^{2}(x, t) d x+\frac{\gamma}{2} \int_{0}^{1} u_{x x}^{2}(x, t) d x .
$$

Suppose, in addition, that $u$ satisfies the initial conditions

$$
\left\{\begin{aligned}
u(x, 0) & =a(x), & & 0<x<1, \\
u_{t}(x, 0) & =b(x), & & 0<x<1 .
\end{aligned}\right.
$$

Given a sequence of initial data $\left\{a^{\epsilon}, b^{\epsilon}\right\}$, with corresponding family of solutions $\left\{u^{\epsilon}\right\}$, we seek to understand how the initial oscillations propagate.

The creation and propagation of oscillations of solutions of (1.1) were studied by Brandon, Fonseca, and Swart [BFS] in the case where local penalization of surface energy is not taken into account, i.e., when $\gamma=0$. It was shown that if initial velocities have oscillations then these are lost immediately as time increases, while oscillatory strains $\left\{u_{x}^{\epsilon}\right\}$ (hence microstructure) cannot be created, but persist for all times if initially present. Here we prove that there is no propagation of oscillations in the velocities and strains; precisely, if $\left\{a^{\epsilon}, b^{\epsilon}\right\}$ remains bounded in $H_{0}^{2}(0,1) \times L^{2}(0,1)$ then for every $T>0,\left\{u^{\epsilon}\right\}$ and $\left\{u_{t}^{\epsilon}\right\}$ converge strongly as $\epsilon \rightarrow 0$ in $L^{2}((0,1) \times(0, T))$, and $\left\{u_{x}^{\epsilon}\right\},\left\{u_{x x}^{\epsilon}\right\}$ converge strongly as $\epsilon \rightarrow 0$ in $L_{\text {loc }}^{2}((0,1) \times(0, T))$ (see Proposition 3.4 and Theorems 3.6, 3.7). Moreover, we show that there is no creation of oscillations in $\left\{u_{x t}^{\epsilon}\right\}$, in that if $\left\{a^{\epsilon}, b^{\epsilon}\right\}$ is bounded in $\left(H_{0}^{2}(0,1) \cap H^{4}(0,1)\right) \times H_{0}^{2}(0,1)$ then $\left\{u_{x t}^{\epsilon}\right\}$ converges strongly in $L_{\text {loc }}^{2}((0,1) \times(0, T))$. These results rely heavily on the theory of compensated compactness of Murat and Tartar (see [M], [T1]), and on Aubin's interpolation theorem (see [A], [L], and [T2]).

In Sec. 2 we establish existence and uniqueness of strong and weak solutions for (1.1)(1.3). In Sec. 3 we study the creation and propagation of oscillations. We end this section remarking that if the Dirichlet boundary conditions were placed on $u_{x x}$ rather than on the strains $u_{x}$, and assuming that $\beta^{2}>4 \gamma$ and that $\sup _{\epsilon}\left\|a^{\epsilon}\right\|_{H^{3}}+\left\|b^{\epsilon}\right\|_{H^{1}}<+\infty$, then the strong convergence of $\left\{u^{\epsilon}\right\},\left\{u_{x}^{\epsilon}\right\},\left\{u_{t}^{\epsilon}\right\},\left\{u_{x x}^{\epsilon}\right\}$ in $L^{2}((0,1) \times(0, T))$ and of $\left\{u_{x t}^{\epsilon}\right\},\left\{u_{x x x}^{\epsilon}\right\}$ in $L_{\text {loc }}^{2}((0,1) \times(0, T))$ for all $T>0$ could be easily obtained using energy a priori estimates (see Proposition 3.11). In Sec. 4 we use a finite difference scheme to illustrate the behavior of solutions in the case where $a^{\epsilon}=0$ and $b^{\epsilon}=\sin ^{6}\left(\frac{1}{\epsilon} \pi x\right)$. In particular, we show that $\left\{u_{x t}^{\epsilon}\right\}$ does not oscillate although the unboundedness of $\left\{b^{\epsilon}\right\}$ in $H^{2}$ prevents us from applying Theorem 3.10 . 
2. Formulation of the problem. Existence theorem. In this section we will obtain existence of solution for the equation

$$
u_{t t}=\left[u_{x}^{3}-u_{x}+\beta u_{x t}-\gamma u_{x x x}\right]_{x}-\alpha u
$$

where $(x, t) \in(0,1) \times(0, \infty), \beta, \gamma>0, \alpha \geq 0$, with boundary conditions

$$
\left\{\begin{array}{cc}
u(0, t)=u(1, t)=0, & t>0, \\
u_{x}(0, t)=u_{x}(1, t)=0, & t>0,
\end{array}\right.
$$

and initial conditions

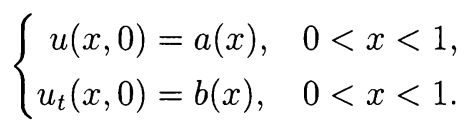

The total energy corresponding to the system is

$$
E(t):=\mathcal{K}(t)+\mathcal{I}(t),
$$

where the kinetic energy is given by

$$
\mathcal{K}(t):=\frac{1}{2} \int_{0}^{1} u_{t}^{2}(x, t) d x
$$

and the potential energy is defined as

$$
\mathcal{I}(t):=\frac{1}{4} \int_{0}^{1}\left(u_{x}^{2}(x, t)-1\right)^{2} d x+\frac{\alpha}{2} \int_{0}^{1} u^{2}(x, t) d x+\frac{\gamma}{2} \int_{0}^{1} u_{x x}^{2}(x, t) d x .
$$

Differentiating the total energy

$$
\begin{aligned}
E(t):= & \frac{1}{2} \int_{0}^{1} u_{t}^{2}(x, t) d x+\frac{1}{4} \int_{0}^{1}\left(u_{x}^{2}(x, t)-1\right)^{2} d x \\
& +\frac{\alpha}{2} \int_{0}^{1} u^{2}(x, t) d x+\frac{\gamma}{2} \int_{0}^{1} u_{x x}^{2}(x, t) d x
\end{aligned}
$$

and using (2.1)-(2.3), we obtain

$$
\frac{d E}{d t}(t)=-\int_{0}^{1} \beta u_{x t}^{2}(x, t) d x
$$

hence $E(\cdot)$ is nonincreasing.

We prove existence and uniqueness of a classical solution for problem (2.1)-(2.3).

Theorem 2.1. Let $a \in H^{4}(0,1) \cap H_{0}^{2}(0,1)$ and $b \in H_{0}^{2}(0,1)$. There exists a unique global solution of $(2.1)-(2.3)$ with $u(t) \in H^{4}(0,1)$ for all $t \geq 0$ and such that

$$
u \in C^{1}\left([0, \infty) ; H_{0}^{2}(0,1)\right) \cap C^{2}\left([0, \infty) ; L^{2}(0,1)\right) .
$$

Proof. We introduce the new variables

$$
\left\{\begin{array}{l}
v:=u_{x x} \\
w:=u_{t}
\end{array}\right.
$$


Then (2.1) and (2.3) can be rewritten as the initial-value problem

$$
\left\{\begin{array}{l}
z_{t}=A z+f(z), \quad t>0 \\
z(0)=z_{0}
\end{array}\right.
$$

in the Banach space $X:=L^{2}(0,1) \times L^{2}(0,1)$, where $z=(v, w), z_{0}=\left(a_{x x}, b\right)$,

$$
A:=\left[\begin{array}{cc}
0 & \Delta \\
-\gamma \Delta & \beta \Delta
\end{array}\right], \quad f(z):=\left[\begin{array}{c}
0 \\
G(v)
\end{array}\right]
$$

$\Delta:=\partial^{2} / \partial x^{2}$, and

$$
G(v(x, t)):=\left(3 V^{2}(x, t)-1\right) v(x, t)-\alpha \int_{0}^{x} V(s, t) d s, \quad V(x, t):=\int_{0}^{x} v(s, t) d s .
$$

The boundary conditions (2.2) imply

$$
\left\{\begin{array}{l}
w(0, t)=w_{x}(0, t)=0 \\
w(1, t)=w_{x}(1, t)=0
\end{array}\right.
$$

We define $D(A):=H^{2}(0,1) \times H_{0}^{2}(0,1)$, and we consider in $X$ the inner product

$$
\left\langle z_{1}, z_{2}\right\rangle:=\int_{0}^{1}\left(\gamma v_{1} v_{2}+w_{1} w_{2}\right) d x
$$

where $z_{i}=\left(v_{i}, w_{i}\right), i=1,2$.

By virtue of (2.4), if $u$ is a solution of (2.1)-(2.3) then

$$
\|z(t)\|_{X}^{2} \leq 2 E(t) \leq 2 E(0)=: M^{2},
$$

and so it is natural to consider the truncated problem

$$
\left\{\begin{array}{l}
z_{t}=A z+\bar{f}(z), \quad t>0 \\
z(0)=z_{0}
\end{array}\right.
$$

where

$$
\bar{f}(z):=\kappa(\|z\|) f(z),
$$

and $\kappa(\cdot) \in C^{1}([0, \infty))$ is a decreasing function such that $\kappa(s)=1$ for $0 \leq s \leq \frac{3 \Lambda}{2}$, and $\kappa(s)=0$ for $s>2 M$. By Theorems 6.1.2 and 6.1.5 in Pazy [P], the initial value problem (2.8) admits a unique solution

$$
\begin{gathered}
(v, w) \in C^{1}\left([0, \infty) ; L^{2}(0,1) \times L^{2}(0,1)\right), \\
(v(t), w(t)) \in H^{2}(0,1) \times H_{0}^{2}(0,1) \text { for all } t \geq 0,
\end{gathered}
$$

provided $A$ generates a $C^{0}$ semigroup on $X, \bar{f}$ is globally Lipschitz and continuously differentiable from $X$ to $X$, and $z_{0} \in D(A)$. We start by showing that $A$ generates a $C^{0}$ semigroup on $X$. By the Hille-Yosida Theorem (see $[\mathrm{P}]$, Theorem 1.3.1), it suffices to prove that

$$
\left\|(\lambda I-A)^{-1}\right\| \leq \frac{1}{\lambda} \text { for all } \lambda>0 .
$$

Fix $\lambda>0$, let $z \in D(A)$, and set $y:=(\lambda I-A) z, y=:(k, h)$. Thus

$$
k=\lambda v-w_{x x}, \quad h=\lambda w+\gamma v_{x x}-\beta w_{x x},
$$


and since $w \in H_{0}^{2}(0,1)$ we have

$$
\begin{aligned}
\|y\|_{X}\|z\|_{X} & \geq\langle y, z\rangle=\int_{0}^{1}(\gamma k v+h w) d x \\
& =\int_{0}^{1}\left[\gamma v\left(\lambda v-w_{x x}\right)+w\left(\lambda w+\gamma v_{x x}-\beta w_{x x}\right)\right] d x \\
& =\lambda \int_{0}^{1}\left(\gamma v^{2}+w^{2}\right) d x+\gamma \int_{0}^{1}\left(-v w_{x x}+w v_{x x}\right) d x-\beta \int_{0}^{1} w w_{x x} d x \\
& =\lambda\|z\|_{X}^{2}+\left.\gamma\left(-v w_{x}+w v_{x}\right)\right|_{0} ^{1}-\left.\beta w w_{x}\right|_{0} ^{1}+\beta \int_{0}^{1} w_{x}^{2} d x \\
& \geq \lambda\|z\|_{X}^{2} .
\end{aligned}
$$

Hence

$$
\|y\|_{X} \geq \lambda\|z\|_{X}
$$

proving (2.9). In order to show that $\bar{f}$ is globally Lipschitz, we assert that

$$
\left\|f\left(z_{1}\right)-f\left(z_{2}\right)\right\|_{X} \leq C\left(1+\left\|z_{1}\right\|_{X}^{2}+\left\|z_{2}\right\|_{X}^{2}\right)\left\|z_{1}-z_{2}\right\|_{X} .
$$

Indeed

$$
\begin{aligned}
& \left\|f\left(z_{1}\right)-f\left(z_{2}\right)\right\|_{X}^{2} \\
& \quad=\int_{0}^{1}\left\{3\left(V_{1}^{2} v_{1}-V_{2}^{2} v_{2}\right)-\left(v_{1}-v_{2}\right)-\alpha \int_{0}^{x}\left(V_{1}(s)-V_{2}(s)\right) d s\right\}^{2} d x \\
& \quad \leq C\left(\int_{0}^{1}\left(V_{1}^{2} v_{1}-V_{2}^{2} v_{2}\right)^{2} d x+\int_{0}^{1}\left(v_{1}-v_{2}\right)^{2} d x+\int_{0}^{1}\left[\int_{0}^{x}\left(V_{1}(s)-V_{2}(s)\right) d s\right]^{2} d x\right) \\
& \quad \leq C\left(\left\|V_{1}^{2} v_{1}-V_{2}^{2} v_{2}\right\|_{L^{2}}^{2}+\left\|v_{1}-v_{2}\right\|_{L^{2}}^{2}+\left\|V_{1}-V_{2}\right\|_{L^{2}}^{2}\right) .
\end{aligned}
$$

Since

$$
\left\|V_{i}\right\|_{\infty} \leq\left\|v_{i}\right\|_{L^{2}}, \quad i=1,2,
$$

we conclude (2.10), and the Lipschitz property for $\bar{f}$ follows immediately from the fact that $\kappa$ is Lipschitz and has compact support. Continuous differentiability of $\bar{f}$ may be easily obtained.

We define

$$
u(x, t):=\int_{0}^{x} \int_{0}^{y} v(z, t) d z d y .
$$

Clearly $u_{x x}=v$, and (2.8) implies that

$$
u_{t}(x, t)=\int_{0}^{x} \int_{0}^{y} v_{t}(z, t) d z d y=\int_{0}^{x} \int_{0}^{y} w_{x x}(z, t) d z d y=w(x, t)
$$

because $w \in H_{0}^{2}(0,1)$. Thus

$$
u_{t}(1, t)=w(1, t)=0, \quad u_{x t}(1, t)=w_{x}(1, t)=0
$$


hence $u(1, \cdot)$ and $u_{x}(1, \cdot)$ are constant functions. Since $a \in H_{0}^{2}(0,1)$ we have

$$
u(1, t)=u(1,0)=\int_{0}^{1} \int_{0}^{x} v(y, 0) d y d x=\int_{0}^{1} \int_{0}^{x} a_{x x}(y) d y d x=0
$$

and

$$
u_{x}(1, t)=u_{x}(1,0)=\int_{0}^{1} a_{x x}(x) d x=0 .
$$

Note that the conditions $u(0, t)=u_{x}(0, t)=0$ follow trivially from (2.12). Moreover, (2.12) and (2.13) yield

$$
u(x, 0)=\int_{0}^{x} \int_{0}^{y} a_{x x}(z) d z d y=a(x), \quad u_{t}(x, 0)=w(x, 0)=b(x) .
$$

By (2.8)

$$
\begin{aligned}
u_{t t}=w_{t} & =\beta w_{x x}-\gamma v_{x x}+\kappa\left(\|z\|_{X}\right) G(v) \\
& =\beta u_{x x t}-\gamma u_{x x x x}+\kappa\left(\|z\|_{X}\right)\left[\left(3 u_{x}^{2}-1\right) u_{x x}-\alpha u\right] .
\end{aligned}
$$

Now

$$
\|z(t)\|_{X}^{2}=\int_{0}^{1}\left(\gamma v^{2}(x, t)+w^{2}(x, t)\right) d x=\int_{0}^{1}\left(u_{t}^{2}(x, t)+\gamma u_{x x}^{2}(x, t)\right) d x
$$

is a continuous function of $t$ with

$$
\|z(0)\|_{X} \leq M
$$

Hence

$$
\mathcal{A}:=\left\{\tau>0:\|z(t)\|_{X} \leq \frac{3}{2} M \text { for all } t \in[0, \tau)\right\}
$$

is nonempty. Let $T$ be the supremum of $\mathcal{A}$. Clearly $\kappa\left(\|z(t)\|_{X}\right)=1$ for all $t \in[0, T)$. Therefore $z$ is a solution of (2.5) (that is, $u$ is a solution of (2.1)-(2.3)) in $[0, T)$ and (2.7) implies the energy bounds obtained earlier, i.e.,

$$
\|z(t)\|_{X}^{2} \leq 2 E(t) \leq 2 E(0) \leq M^{2}
$$

for all $t<T$. If $T$ was finite then, by continuity, $\|z(T)\|_{X} \leq M$ and so $\|z(t)\|_{X} \leq \frac{3 M I}{2}$ for $t \in\left[0, T^{*}\right)$ for some $T^{*}>T$, contradicting the fact that $T$ is the supremum of $\mathcal{A}$. The regularity properties of $u$ stated in the theorem follow from (2.12) and (2.13).

Existence of weak solutions under milder initial regularity assumptions is guaranteed by Theorem 6.1.2 in Pazy [P].

Theorem 2.2. Let $a \in H_{0}^{2}(0,1)$ and $b \in L^{2}(0,1)$. There exist unique weak solutions $u$ of $(2.1)-(2.3)$, and $z=(v, w)$ of $(2.8)$, with

$$
\begin{gathered}
u \in C\left([0, \infty) ; H_{0}^{2}(0,1)\right) \cap C^{1}\left([0, \infty) ; L^{2}(0,1)\right), \\
(v, w) \in C\left([0, \infty) ; L^{2}(0,1) \times L^{2}(0,1)\right) .
\end{gathered}
$$


3. Propagation and creation of oscillations. Consider the parametrized family of problems

$$
u_{t t}^{\epsilon}=\left[\left(u_{x}^{\epsilon}\right)^{3}-u_{x}^{\epsilon}+\beta u_{x t}^{\epsilon}-\gamma u_{x x x}^{\epsilon}\right]_{x}-\alpha u^{\epsilon}
$$

with boundary conditions (2.2) and initial conditions

$$
\begin{cases}u^{\epsilon}(x, 0)=a^{\epsilon}(x), & 0<x<1, \\ u_{t}^{\epsilon}(x, 0)=b^{\epsilon}(x), & 0<x<1 .\end{cases}
$$

We assume that

$$
\left\|a^{\epsilon}\right\|_{H^{2}}+\left\|b^{\epsilon}\right\|_{L^{2}} \leq C
$$

where $C$ does not depend on $\epsilon$.

In the sequel $C$ will denote a generic positive constant that may vary from formula to formula. If $X$ is a Banach space and $0<T \leq+\infty$ then a sequence $w^{\epsilon}:(0, T) \rightarrow X$ of Borel measurable functions is said to belong to $B L^{2}(0, T ; X)$ if

$$
\sup _{\epsilon} \int_{0}^{T}\left\|w^{\epsilon}(t)\right\|_{X}^{2} d t \leq C
$$

for some constant $C>0$. Also, we write $\left\{w^{\epsilon}\right\} \in B L^{\infty}(0, T ; X)$ if

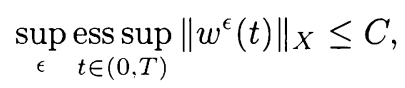

and $\left\{w^{\epsilon}\right\} \in B H^{1}(0, T ; X)$ if

$$
\sup _{\epsilon}\left\{\int_{0}^{T}\left\|w^{\epsilon}(t)\right\|_{X}^{2} d t+\int_{0}^{T}\left\|w_{t}^{\epsilon}(t)\right\|_{X}^{2} d t\right\} \leq C .
$$

By virtue of the bounds (3.1), the initial sequence of strains $\left\{a_{x}^{\epsilon}\right\}$ does not oscillate. We start by showing that oscillations are not created in $\left\{u_{x}^{\epsilon}\right\}$ for $t>0$. To fix notation, if $0<T<+\infty$ we define $\mathcal{K}_{T}:=(0,1) \times(0, T)$ and $\mathcal{K}_{\infty}:=(0,1) \times(0, \infty)$.

Proposition 3.1. Assume that (3.1) holds. Then $\left\{u^{\epsilon}\right\} \in B L^{\infty}\left(0, \infty ; H_{0}^{2}(0,1)\right),\left\{u_{t}^{\epsilon}\right\} \in$ $B L^{\infty}\left(0, \infty, L^{2}(0,1)\right)$, and $\left\{u_{x t}^{\epsilon}\right\} \in B L^{2}\left(\mathcal{K}_{T}\right)$. In particular, there exists a subsequence and $U \in L^{\infty}\left(0, \infty ; H_{0}^{2}(0,1)\right)$ such that

$$
\begin{gathered}
u^{\epsilon}-U \text { weakly* in } L^{\infty}\left(0, \infty ; H_{0}^{2}(0,1)\right), \\
u_{t}^{\epsilon}-U_{t} \text { weakly* in } L^{\infty}\left(0, \infty ; L^{2}(0,1)\right), \\
u_{t x}^{\epsilon} \rightarrow U_{t x} \text { weakly in } L^{2}\left(\mathcal{K}_{\infty}\right) .
\end{gathered}
$$

Proof. We note that (2.4) implies that

$$
\begin{aligned}
E^{\epsilon}(t)= & \frac{1}{2} \int_{0}^{1}\left(u_{t}^{\epsilon}\right)^{2}(x, t) d x+\frac{1}{4} \int_{0}^{1}\left(\left(u_{x}^{\epsilon}\right)^{2}(x, t)-1\right)^{2} d x \\
& +\frac{\alpha}{2} \int_{0}^{1}\left(u^{\epsilon}\right)^{2}(x, t) d x+\frac{\gamma}{2} \int_{0}^{1}\left(u_{x x}^{\epsilon}\right)^{2}(x, t) d x \\
\leq & E^{\epsilon}(0) \leq C .
\end{aligned}
$$


Thus

$$
\left\{u^{\epsilon}\right\} \in B L^{\infty}\left(0, \infty ; H_{0}^{2}(0,1)\right),\left\{u_{t}^{\epsilon}\right\} \in B L^{\infty}\left(0, \infty ; L^{2}(0,1)\right) .
$$

Again, (2.4) implies that for all $\tau>0$

$$
C \geq E^{\epsilon}(0) \geq E^{\epsilon}(0)-E^{\epsilon}(\tau)=\beta \int_{0}^{\tau} \int_{0}^{1}\left(u_{x t}^{\epsilon}\right)^{2}(x, t) d x d t
$$

and so, letting $\tau \rightarrow \infty$ we conclude that $\left\{u_{x t}^{\epsilon}\right\} \in B L^{2}\left(\mathcal{K}_{\infty}\right)$.

In what follows we assume that a subsequence has been extracted in accordance with the previous result. The strong convergence of $\left\{u_{x}^{\epsilon}\right\}$ is provided by the method of compensated compactness ([M], [T1]), more precisely by the Div-Curl Lemma (see Theorem 1.1 in [T1]).

Lemma 3.2. Let $\Omega \subset \mathbf{R}^{N}$ be an open, bounded domain, and let $E^{\epsilon}, G^{\epsilon} \in L^{2}\left(\Omega ; \mathbf{R}^{N}\right)$ be such that

$$
\sum_{i=1}^{N} \frac{\partial E_{i}^{\epsilon}}{\partial x_{i}}, \quad \frac{\partial G_{i}^{\epsilon}}{\partial x_{j}}-\frac{\partial G_{j}^{\epsilon}}{\partial x_{i}}
$$

belong to a compact subset of $H_{\mathrm{loc}}^{-1}$, for all $1 \leq i, j \leq N$. Suppose further that $E^{\epsilon} \rightarrow E_{0}$ and $G^{\epsilon} \rightarrow G_{0}$ in $L^{2}\left(\Omega ; \mathbf{R}^{N}\right)$. Then

$$
E^{\epsilon} \cdot G^{\epsilon}:=\sum_{i=1}^{N} E_{i}^{\epsilon} G_{i}^{\epsilon} \rightarrow E_{0} \cdot G_{0}
$$

in the sense of distributions.

An immediate consequence is

Corollary 3.3. Fix $T>0$ and let $\left\{U^{\epsilon}\right\}$ be a bounded sequence in $H^{1}\left(\mathcal{K}_{T}\right)$ such that $\left\{U_{x x}^{\epsilon}\right\}$ is bounded in $L^{2}\left(\mathcal{K}_{T}\right)$. If $U^{\epsilon} \rightarrow U$ weakly in $H^{1}\left(\mathcal{K}_{T}\right)$ then $U_{x}^{\epsilon} \rightarrow U_{x}$ strongly in $L_{\text {loc }}^{2}\left(\mathcal{K}_{T}\right)$.

Proof. Using the notation

$$
\operatorname{div}(f, g):=f_{x}+g_{t}, \quad \operatorname{curl}(f, q):=f_{t}-g_{x},
$$

it follows that $E^{\epsilon}:=\left(U_{x}^{\epsilon}, 0\right) \rightarrow E_{0}:=\left(U_{x}, 0\right)$ and $G^{\epsilon}:=\left(U_{x}^{\epsilon}, U_{t}^{\epsilon}\right) \rightarrow G_{0}:=\left(U_{x}, U_{t}\right)$ weakly in $L^{2}\left(\mathcal{K}_{T}\right)$, with

$$
\operatorname{div}\left(U_{x}^{\epsilon}, 0\right) \in B L^{2}\left(\mathcal{K}_{T}\right) \text { and } \operatorname{curl}\left(U_{x}^{\epsilon}, U_{t}^{\epsilon}\right)=0 .
$$

Therefore, by the Div-Curl Lemma (see Lemma 3.2)

$$
\left(U_{x}^{\epsilon}\right)^{2} \rightarrow\left(U_{x}\right)^{2}
$$

in the sense of distributions. Hence, for every $\phi \in D\left(\mathcal{K}_{T}\right)$ we have

$$
\begin{aligned}
\int_{\mathcal{K}_{\tau}}\left(U_{x}^{\epsilon}-U_{x}\right)^{2} \phi= & \int_{\mathcal{K}_{\tau}}\left(U_{x}^{\epsilon}\right)^{2} \phi-2 U_{x}^{\epsilon} U_{x} \phi+\left(U_{x}\right)^{2} \phi \\
& \rightarrow \int_{\mathcal{K}_{\tau}}\left(U_{x}\right)^{2} \phi-2\left(U_{x}\right)^{2} \phi+\left(U_{x}\right)^{2} \phi=0,
\end{aligned}
$$


and we conclude that

$$
U_{x}^{\epsilon} \rightarrow U_{x} \text { strongly in } L_{\text {loc }}^{2}\left(\mathcal{K}_{T}\right) \text {. }
$$

Note that by Proposition 3.1 the sequence $U^{\epsilon}:=u^{\epsilon}$ is bounded in $H^{1}\left(\mathcal{K}_{\infty}\right)$ and it satisfies the hypotheses of Corollary 3.3. Using the Sobolev-Rellich-Kondrachov Compact Imbedding Theorem and Corollary 3.3 we obtain the following result.

Proposition 3.4. Under the hypotheses of Proposition 3.1, $u^{\epsilon} \rightarrow U$ strongly in $L^{p}\left(\mathcal{K}_{T}\right)$ and $u_{x}^{\epsilon} \rightarrow U_{x}$ strongly in $L_{\text {loc }}^{p}\left(\mathcal{K}_{\infty}\right)$ for all $1 \leq p<\infty$.

Strong convergence of $\left\{u_{t}^{\epsilon}\right\}$ is an immediate consequence of an interpolation compactness result due to Aubin (see [A], [L], Theorem 5.1, and [T2], p. 50).

Theorem 3.5. Let $B_{0} \subset B_{1} \subset B_{2}$ be three Hilbert spaces with $B_{1}$ continuously imbedded in $B_{2}$ and $B_{0}$ compactly imbedded in $B_{1}$. Let $1<p<\infty$ and consider a sequence $\left\{V^{\epsilon}\right\}$ such that

$$
V^{\epsilon} \in B L^{p}\left(0, T ; B_{0}\right), \quad \frac{d V^{\epsilon}}{d t} \in B L^{p}\left(0, T ; B_{2}\right) .
$$

Then $\left\{V^{\epsilon}\right\}$ is contained in a compact subset of $L^{p}\left(0, T ; B_{1}\right)$.

We have

THEOREM 3.6. Under the hypotheses of Proposition 3.1

$$
u_{t}^{\epsilon} \rightarrow U_{t} \text { strongly in } L^{2}\left(\mathcal{K}_{T}\right) \text { for all } T>0 .
$$

Proof. Set

$$
V^{\epsilon}:=u_{t}^{\epsilon}
$$

By Proposition 3.1 we have

$$
V^{\epsilon} \in B L^{2}\left(0, T ; H_{0}^{1}(0,1)\right),
$$

and using (2.1) we obtain

$$
\frac{d V^{\epsilon}}{d t}=u_{t t}^{\epsilon} \in B L^{2}\left(0, T ; H^{-2}(0,1)\right) .
$$

Setting $B_{0}:=H_{0}^{1}(0,1), B_{1}:=L^{2}(0,1)$, and $B_{2}:=H^{-2}(0,1)$, from Theorem 3.5 we conclude that every subsequence of $\left\{u_{t}^{\epsilon}\right\}$ admits a subsequence converging strongly in $L^{2}\left(\mathcal{K}_{T}\right)$, and so

$$
u_{t}^{\epsilon} \rightarrow U_{t} \text { strongly in } L^{2}\left(\mathcal{K}_{T}\right) .
$$

Next we want to show that

TheOREM 3.7. Under the hypotheses of Proposition 3.1,

$$
u_{x x}^{\epsilon} \rightarrow U_{x x} \text { strongly in } L_{\text {loc }}^{2}\left(\mathcal{K}_{\infty}\right) \text {. }
$$

This result will follow from the partial differential equations satisfied by $u^{\epsilon}$ and from the compactness lemma below. 
Lemma 3.8. Fix $T>0$ and let $\left\{R^{\epsilon}\right\},\left\{U^{\epsilon}\right\},\left\{V^{\epsilon}\right\},\left\{W^{\epsilon}\right\},\left\{Y^{\epsilon}\right\},\left\{Z^{\epsilon}\right\} \in B L^{2}\left(\mathcal{K}_{T}\right)$. Suppose further that

$$
\left\{\begin{aligned}
U_{x x}^{\epsilon} & =R_{x}^{\epsilon}+V_{t}^{\epsilon}+W^{\epsilon}, \\
U_{t}^{\epsilon} & =Z_{x}^{\epsilon}+Y^{\epsilon} .
\end{aligned}\right.
$$

Then $\left\{U^{\epsilon}\right\}$ remains in a bounded subset of $H^{1 / 3}\left(\mathcal{K}^{\prime}\right)$ for every compact $\mathcal{K}^{\prime} \Subset \mathcal{K}_{T}$.

Before proving this result we show how to derive Theorem 3.7.

Proof of Theorem 3.7. Set $U^{\epsilon}:=u_{x x}^{\epsilon}$. By Proposition 3.1 we have $\left\{U^{\epsilon}\right\} \in B L^{2}\left(\mathcal{K}_{T}\right)$,

$$
U_{t}^{\epsilon}=Z_{x}^{\epsilon}, \text { where } Z^{\epsilon}:=u_{x t}^{\epsilon} \in B L^{2}\left(\mathcal{K}_{T}\right),
$$

and

$$
\begin{aligned}
U_{x x}^{\epsilon}=u_{x x x x}^{\epsilon} & =\frac{1}{\gamma}\left[\left(\left(u_{x}^{\epsilon}\right)^{3}-u_{x}^{\epsilon}\right)_{x}+\beta u_{x x t}^{\epsilon}-\alpha u^{\epsilon}-u_{t t}^{\epsilon}\right] \\
& =R_{x}^{\epsilon}+V_{t}^{\epsilon}+W^{\epsilon}
\end{aligned}
$$

where

$$
R^{\epsilon}:=\frac{1}{\gamma}\left(\left(u_{x}^{\epsilon}\right)^{3}-u_{x}^{\epsilon}\right), \quad V^{\epsilon}:=\frac{1}{\gamma}\left(\beta u_{x x}^{\epsilon}-u_{t}^{\epsilon}\right), \quad W^{\epsilon}:=-\frac{\alpha}{\gamma} u^{\epsilon} .
$$

By the Sobolev Imbedding Theorem and Proposition 3.1 it follows that $\left\{R^{\epsilon}\right\},\left\{V^{\epsilon}\right\}$, and $\left\{W^{\epsilon}\right\} \in B L^{2}\left(\mathcal{K}_{T}\right)$. Hence, due to Lemma 3.8 we have that $\left\{u_{x x}^{\epsilon}\right\}$ is in a bounded subset of $H^{1 / 3}\left(\mathcal{K}^{\prime}\right)$ and, in particular, $\left\{u_{x x}^{\epsilon}\right\}$ is in a compact set of $L_{\text {loc }}^{2}\left(\mathcal{K}^{\prime}\right)$ for every compact $\mathcal{K}^{\prime} \Subset \mathcal{K}_{T}$.

Proof of Lemma 3.8. Fix $\varphi \in C_{0}^{\infty}(0, T), \psi \in C_{0}^{\infty}(0,1)$ and define

$$
\widetilde{U}^{\epsilon}:=\varphi(t) \psi(x) U^{\epsilon}(x, t) .
$$

Then

$$
\left\{\begin{aligned}
\widetilde{U}_{x x}^{\epsilon} & =A_{x}^{\epsilon}+B_{t}^{\epsilon}+C^{\epsilon}, \\
\widetilde{U}_{t}^{\epsilon} & =D_{x}^{\epsilon}+E^{\epsilon}
\end{aligned}\right.
$$

where

$$
A^{\epsilon}:=2 \varphi \psi_{x} U^{\epsilon}+\varphi \psi R^{\epsilon}, \quad B^{\epsilon}:=\varphi \psi V^{\epsilon}, \quad C^{\epsilon}:=-\varphi \psi_{x x} U^{\epsilon}+\varphi \psi W^{\epsilon}-\varphi_{t} \psi V^{\epsilon}-\varphi \psi_{x} R^{\epsilon}
$$

and

$$
D^{\epsilon}:=\varphi \psi Z^{\epsilon}, \quad E^{\epsilon}:=\varphi_{t} \psi U^{\epsilon}-\varphi \psi_{x} Z^{\epsilon}+\varphi \psi U^{\epsilon}
$$

are uniformly bounded in $L^{2}\left(\mathcal{K}_{T}\right)$. Applying a Fourier transform to the system of equations we have

$$
\left\{\begin{array}{c}
-\xi^{2} \hat{\widetilde{U}}^{\epsilon}=i \xi \hat{A}^{\epsilon}+i \tau \hat{B}^{\epsilon}+\hat{C}^{\epsilon} \\
i \tau \tilde{\widetilde{U}}^{\epsilon}=i \xi \hat{D}^{\epsilon}+\hat{E}^{\epsilon} .
\end{array}\right.
$$

Therefore

$$
\hat{\tilde{U}}^{\epsilon} \frac{|\xi|^{2}}{|\xi|+|\tau|+1}, \quad \hat{\tilde{U}}^{\epsilon} \frac{|\tau|}{|\xi|+1} \in B L^{2}\left(\mathbf{R}^{2}\right)
$$


and since $\hat{\widetilde{U}}^{\epsilon} \in B L^{2}\left(\mathbf{R}^{2}\right)$, from $(3.2)$ we deduce that

$$
\hat{\tilde{U}}^{\epsilon} \frac{|\xi|^{2}+|\xi|+|\tau|+1}{|\xi|+|\tau|+1}, \quad \hat{\tilde{U}} \frac{|\tau|+|\xi|+1}{|\xi|+1} \in B L^{2}\left(\mathbf{R}^{2}\right) .
$$

In particular, using the Hölder inequality with $p=3 / 2$ and $p^{\prime}=3$, we deduce that

$$
\left|\hat{\tilde{U}}^{\epsilon}\right|\left(\frac{|\tau|+|\xi|+1}{|\xi|+1}\right)^{2 / 3}\left(\frac{|\xi|^{2}+|\xi|+|\tau|+1}{|\xi|+|\tau|+1}\right)^{1 / 3} \in B L^{2}\left(\mathbf{R}^{2}\right)
$$

so

$$
\left|\hat{\tilde{U}}^{\epsilon}\right|(|\tau|+|\xi|+1)^{1 / 3}
$$

remains in a bounded set of $L^{2}\left(\mathbf{R}^{2}\right)$. We conclude that $U^{\epsilon}$ is in a bounded set of $H^{1 / 3}\left(\mathcal{K}^{\prime}\right)$ for all compact sets $\mathcal{K}^{\prime} \Subset \mathcal{K}_{T}$.

Next we show that $\left\{u_{x t}^{\epsilon}\right\}$ converges locally strongly provided that the initial data $\left\{\left(a^{\epsilon}, b^{\epsilon}\right)\right\}$ admits uniform bounds on $H^{4}(0,1) \times H^{2}(0,1)$. We remark that this regularity was prescribed in Theorem 2.1 asserting existence of strong solutions, and that under this assumption $\left\{b_{x}^{\epsilon}\right\}$ does not oscillate. We will, therefore, establish that there is no creation of oscillations in $\left\{u_{x t}^{\epsilon}\right\}$. First we provide uniform bounds on $\left\{u_{t t}^{\epsilon}\right\}$ and $\left\{u_{x x t}^{\epsilon}\right\}$.

Proposition 3.9. If $\left\{\left(a^{\epsilon}, b^{\epsilon}\right)\right\}$ is bounded in $\left(H_{0}^{2}(0,1) \cap H^{4}(0,1)\right) \times H_{0}^{2}(0,1)$ then

$$
\left\{u_{t t}^{\epsilon}\right\},\left\{u_{x x t}^{\epsilon}\right\} \in B L^{\infty}\left(0, \infty ; L^{2}(0,1)\right) .
$$

Proof. Let $u^{\epsilon}$ be the solution provided by Theorem 2.1 with initial conditions $\left(a^{\epsilon}, b^{\epsilon}\right)$, and define

$$
\mathcal{E}^{\epsilon}(t):=\frac{1}{2} \int_{0}^{1}\left[\left(u_{t t}^{\epsilon}\right)^{2}(x, t)+\gamma\left(u_{x x t}^{\epsilon}\right)^{2}(x, t)+\alpha\left(u_{t}^{\epsilon}\right)^{2}(x, t)\right] d x .
$$

Note that $\mathcal{E}^{\epsilon}(t)$ is well defined for all $t \geq 0$ due to the fact that $u_{t t}^{\epsilon}, u_{x x t}^{\epsilon}, u_{t}^{\epsilon} \in C([0, \infty)$; $\left.L^{2}(0,1)\right)$.

Define

$$
\Delta_{h} f(x, t):=\frac{f(x, t+h)-f(x)}{h}
$$

By (2.1) we have

$$
\Delta_{h} u_{t t}^{\epsilon}(x, t)=\Delta_{h}\left(\left(u_{x}^{\epsilon}\right)^{3}-u_{x}^{\epsilon}\right)_{x}+\beta \Delta_{h} u_{x x t}^{\epsilon}-\gamma \Delta_{h} u_{x x x x}^{\epsilon}-\alpha \Delta_{h} u^{\epsilon},
$$

and the boundary conditions (2.2) imply that for $t>0$

$$
\Delta_{h} u^{\epsilon}(x, t)=\Delta_{h} u_{x}^{\epsilon}(x, t)=\Delta_{h} u_{t}^{\epsilon}(x, t)=\Delta_{h} u_{x t}^{\epsilon}(x, t)=0 \text { for } x \in\{0,1\} .
$$

Integrating by parts we have

$$
\begin{aligned}
& \int_{0}^{1} \Delta_{h} u_{t t}^{\epsilon}(x, t) \Delta_{h} u_{t}^{\epsilon}(x, t) d x=\int_{0}^{1} \Delta_{h} u_{t}^{\epsilon}(x, t) \Delta_{h}\left(\left(u_{x}^{\epsilon}\right)^{3}-u_{x}^{\epsilon}\right)_{x}(x, t) d x \\
& \quad-\beta \int_{0}^{1}\left(\Delta_{h} u_{x t}^{\epsilon}(x, t)\right)^{2} d x-\gamma \int_{0}^{1} \Delta_{h} u_{x x t}^{\epsilon}(x, t) \Delta_{h} u_{x x}^{\epsilon}(x, t) d x \\
& \quad-\alpha \int_{0}^{1} \Delta_{h} u_{t}^{\epsilon}(x, t) \Delta_{h} u^{\epsilon}(x, t) d x
\end{aligned}
$$


Setting

$$
\mathcal{E}_{h}^{\epsilon}(t):=\frac{1}{2} \int_{0}^{1}\left(\Delta_{h} u_{t}^{\epsilon}\right)^{2}(x, t)+\gamma\left(\Delta_{h} u_{x x}^{\epsilon}\right)^{2}(x, t)+\alpha\left(\Delta_{h} u^{\epsilon}\right)^{2}(x, t) d x,
$$

(3.4) implies that

$$
\begin{aligned}
\frac{d \mathcal{E}_{h}^{\epsilon}(t)}{d t} & =\int_{0}^{1}\left[\Delta_{h} u_{t}^{\epsilon}(x, t) \Delta_{h}\left(\left(u_{x}^{\epsilon}\right)^{3}-u_{x}^{\epsilon}\right)_{x}(x, t)-\beta\left(\Delta_{h} u_{x t}^{\epsilon}(x, t)\right)^{2}\right] d x \\
& =-\int_{0}^{1}\left[\Delta_{h} u_{x t}^{\epsilon}(x, t) \Delta_{h}\left(\left(u_{x}^{\epsilon}\right)^{3}-u_{x}^{\epsilon}\right)(x, t)+\beta\left(\Delta_{h} u_{x t}^{\epsilon}(x, t)\right)^{2}\right] d x
\end{aligned}
$$

Hence

$$
\begin{aligned}
\mathcal{E}_{h}^{\epsilon}(t)=\mathcal{E}_{h}^{\epsilon}(0) & -\int_{0}^{t} \int_{0}^{1} \Delta_{h} u_{x t}^{\epsilon}(x, s) \Delta_{h}\left(\left(u_{x}^{\epsilon}\right)^{3}-u_{x}^{\epsilon}\right)(x, s) d x d s \\
& -\beta \int_{0}^{t} \int_{0}^{1}\left(\Delta_{h} u_{x t}^{\epsilon}(x, s)\right)^{2} d x d s,
\end{aligned}
$$

and since

$$
a x-\beta x^{2} \leq \frac{1}{4 \beta} a^{2} \text { for all } x
$$

we conclude that

$$
\mathcal{E}_{h}^{\epsilon}(t) \leq \mathcal{E}_{h}^{\epsilon}(0)+\frac{1}{4 \beta} \int_{0}^{t} \int_{0}^{1}\left(\Delta_{h}\left(\left(u_{x}^{\epsilon}\right)^{3}-u_{x}^{\epsilon}\right)\right)^{2} d x d s .
$$

Given $f \in C^{1}((0,1) \times(0, t+h))$ we have

$$
\left(\frac{f(x, s+h)-f(x, s)}{h}\right)^{2}=\left(\frac{1}{h} \int_{s}^{s+h} f_{t}(x, r) d r\right)^{2} \leq \frac{1}{h} \int_{s}^{s+h} f_{t}^{2}(x, r) d r
$$

and so

$$
\begin{aligned}
\int_{0}^{t} \int_{0}^{1}\left(\Delta_{h} f\right)^{2}(x, s) d x d s & \leq \frac{1}{h} \int_{0}^{t} \int_{0}^{1} \int_{s}^{s+h} f_{t}^{2}(x, r) d r d x d s \\
& =\frac{1}{h} \int_{0}^{t+h} \int_{0}^{1} \int_{\max \{0, r-h\}}^{\min \{r, t\}} d s f_{t}^{2}(x, r) d x d r \\
& \leq \int_{0}^{t+h} \int_{0}^{1} f_{t}^{2}(x, r) d x d r
\end{aligned}
$$

Using a density argument, we may apply this inequality to $f:=\left(u_{x}^{\epsilon}\right)^{3}-u_{x}^{\epsilon}$, and (3.6) yields

$$
\begin{aligned}
\mathcal{E}_{h}^{\epsilon}(t) & \leq \mathcal{E}_{h}^{\epsilon}(0)+\frac{1}{4 \beta} \int_{0}^{t+h} \int_{0}^{1}\left[\left(\left(u_{x}^{\epsilon}\right)^{3}-u_{x}^{\epsilon}\right)_{t}\right]^{2}(x, r) d x d r \\
& \leq \mathcal{E}_{h}^{\epsilon}(0)+C \beta^{-1}
\end{aligned}
$$

where $C$ is independent of $h, \epsilon$ and $t$, and where we used Proposition 3.1 and the Sobolev Imbedding Theorem to guarantee that $\left\{u_{x}^{\epsilon}\right\} \in B L^{\infty}\left(\mathcal{K}_{\infty}\right)$. Finally, by Theorem 2.1

$$
u_{t}^{\epsilon}, u_{x x}^{\epsilon}, u^{\epsilon} \in C^{1}\left([0, \infty) ; L^{2}(0,1)\right) .
$$


Hence

$$
\left\|\Delta_{h} u_{t}^{\epsilon}-u_{t t}^{\epsilon}\right\|_{L^{2}(0,1)}, \quad\left\|\Delta_{h} u_{x x}^{\epsilon}-u_{x x t}^{\epsilon}\right\|_{L^{2}(0,1)}, \quad\left\|\Delta_{h} u^{\epsilon}-u^{\epsilon}\right\|_{L^{2}(0,1)}
$$

tend to zero as $h \rightarrow 0$, and so

$$
\lim _{h \rightarrow 0} \mathcal{E}_{h}^{\epsilon}(t)=\mathcal{E}^{\epsilon}(t)
$$

Using the fact that the bounds on the initial conditions imply that $\mathcal{E}_{h}^{\epsilon}(0)$ remain bounded, taking the limit in (3.7) as $h \rightarrow 0$ we deduce that

$$
\mathcal{E}^{\epsilon}(t) \leq C\left(1+\frac{1}{\beta}\right) \quad \text { for all } t>0 \text {. }
$$

Finally, we prove

ThEOREM 3.10. If $\left\{\left(a^{\epsilon}, b^{\epsilon}\right)\right\}$ is bounded in $\left(H_{0}^{2}(0,1) \cap H^{4}(0,1)\right) \times H_{0}^{2}(0,1)$ then

$$
u_{x t}^{\epsilon} \rightarrow U_{x t} \text { strongly in } L_{\text {loc }}^{2}\left(\mathcal{K}_{T}\right) \text { for all } T>0 \text {. }
$$

Proof. By Propositions 3.1 and 3.9 the sequence $U^{\epsilon}:=u_{t}^{\epsilon}$ satisfies the hypotheses of Corollary 3.3 and the proof is concluded.

We remark that the proofs leading to strong convergence of $\left\{u^{\epsilon}\right\},\left\{u_{x}^{\epsilon}\right\},\left\{u_{t}^{\epsilon}\right\},\left\{u_{x x}^{\epsilon}\right\}$, and $\left\{u_{x t}^{\epsilon}\right\}$ used different analytical arguments, including the Sobolev-Rellich-Kondrachov Compact Imbedding Theorem, interpolation theorems, Fourier transforms, compensated compactness, and difference quotient regularity techniques. However, we note that if we prescribe Dirichlet boundary conditions on $u_{x x}$ rather than on the strains $u_{x}$, and assuming that $\beta^{2}>4 \gamma$ and that

$$
\sup _{\epsilon}\left\|a^{\epsilon}\right\|_{H^{3}}+\left\|b^{\epsilon}\right\|_{H^{1}}=C<+\infty
$$

then strong convergence of $\left\{u^{\epsilon}\right\},\left\{u_{x}^{\epsilon}\right\},\left\{u_{t}^{\epsilon}\right\},\left\{u_{x x}^{\epsilon}\right\}$ in $L^{2}\left(\mathcal{K}_{T}\right)$ and of $\left\{u_{x t}^{\epsilon}\right\},\left\{u_{x x x}^{\epsilon}\right\}$ in $L_{\text {loc }}^{2}\left(\mathcal{K}_{T}\right)$ for all $T>0$ may be easily obtained using simple energy a priori estimates, compact imbedding theorems and compensated compactness. Indeed, suppose that

$$
u^{\epsilon} \in C\left([0,+\infty) ; H^{3}(0,1)\right) \cap C^{1}\left([0,+\infty) ; H^{1}(0,1)\right) \cap C^{2}\left([0,+\infty) ; H^{-1}(0,1)\right)
$$

is a solution of

$$
u_{t t}^{\epsilon}=\left[\left(u_{x}^{\epsilon}\right)^{3}-u_{x}^{\epsilon}+\beta u_{x t}^{\epsilon}-\gamma u_{x x x}^{\epsilon}\right]_{x}-\alpha u^{\epsilon}
$$

satisfying the boundary conditions

$$
u^{\epsilon}=u_{x x}^{\epsilon}=0 \text { if } x \in\{0,1\}
$$

and the initial conditions

$$
u^{\epsilon}(x, 0)=a^{\epsilon}(x), \quad u_{t}^{\epsilon}(x, 0)=b^{\epsilon}(x) .
$$

Proposition 3.11. For $T>0$ the sequences $\left\{u^{\epsilon}\right\},\left\{u_{x}^{\epsilon}\right\},\left\{u_{t}^{\epsilon}\right\}$, and $\left\{u_{x x}^{\epsilon}\right\}$ converge strongly in $L^{2}\left(\mathcal{K}_{T}\right)$, and $\left\{u_{x t}^{\epsilon}\right\},\left\{u_{x x x}^{\epsilon}\right\}$ converge strongly in $L_{\text {loc }}^{2}\left(\mathcal{K}_{T}\right)$.

As before, using the Sobolev-Rellich-Kondrachov Compact Imbedding Theorem and Corollary 3.3, the above result follows immediately if we prove that

$$
\int_{0}^{T}\left(\left\|u_{x x}^{\epsilon}(t)\right\|_{H^{2}(0,1)}^{2}+\left\|u_{x x t}^{\epsilon}(t)\right\|_{L^{2}(0.1)}^{2}+\left\|u_{t}^{\epsilon}(t)\right\|_{H^{2}(0,1)}^{2}+\left\|u_{t t}^{\epsilon}(t)\right\|_{L^{2}(0,1)}^{2}\right) d t \leq C .
$$


To prove this inequality first we remark that by virtue of (2.4), which still holds, we have

$$
E^{\epsilon}(t) \leq E^{\epsilon}(0) \leq C
$$

and so

$$
\left\|u_{t}^{\epsilon}(t)\right\|_{L^{2}(0,1)}+\left\|u_{x x}^{\epsilon}(t)\right\|_{L^{2}(0,1)} \leq C .
$$

We recall the following regularity result (see [E], p. 326). If $z \in L^{2}\left(0, T ; H_{0}^{1}(0,1)\right)$, $z_{t} \in L^{2}\left(0, T ; H^{-1}(0,1)\right)$, and if

$$
z_{t}=\mu z_{x x}+F
$$

where $\mu>0, F \in L^{2}\left(0, T ; L^{2}(0,1)\right)$, and $z(\cdot, 0) \in H_{0}^{1}(0,1)$, then

$$
\begin{aligned}
& \int_{0}^{T}\left(\|z(t)\|_{H^{2}(0.1)}^{2}+\left\|z_{t}(t)\right\|_{L^{2}(0.1)}^{2}\right) d t \\
& \quad \leq C\left(\|z(0)\|_{H_{0}^{1}(0.1)}^{2}+\int_{0}^{T}\|F(t)\|_{L^{2}(0.1)}^{2} d t\right) .
\end{aligned}
$$

We may write (3.9) as

$$
\left(\partial_{t}-A \partial_{x}^{2}\right)\left(\partial_{t}-B \partial_{x}^{2}\right) u^{\epsilon}=-\alpha u^{\epsilon}+\left(\left(u_{x}^{\epsilon}\right)^{3}-u_{x}^{\epsilon}\right)_{x},
$$

where $A, B>0$, and setting $w^{\epsilon}:=\left(\partial_{t}-B \partial_{x}^{2}\right) u^{\epsilon}$ and $f^{\epsilon}:=-\alpha u^{\epsilon}+\left(\left(u_{x}^{\epsilon}\right)^{3}-u_{x}^{\epsilon}\right)_{x}$ we deduce that

$$
w_{t}^{\epsilon}=A w_{x x}^{\epsilon}+f^{\epsilon}, \quad \text { and } \quad w^{\epsilon}=0 \text { if } x \in\{0,1\} .
$$

By (3.8), (3.11), and (3.12) we have

$$
\begin{aligned}
& \int_{0}^{T}\left(\left\|w^{\epsilon}(t)\right\|_{H^{2}(0,1)}^{2}+\left\|w_{t}^{\epsilon}(t)\right\|_{L^{2}(0,1)}^{2}\right) d t \\
& \quad \leq C\left(\left\|b^{\epsilon}-B a_{x x}^{\epsilon}\right\|_{H_{0}^{1}(0,1)}^{2}+\int_{0}^{T}\left\|f^{\epsilon}(t)\right\|_{L^{2}(0.1)}^{2} d t\right) \\
& \quad \leq C .
\end{aligned}
$$

Since

$$
u_{t}^{\epsilon}=B u_{x x}^{\epsilon}+w^{\epsilon}
$$

letting $z^{\epsilon}:=u_{t}^{\epsilon}$ or $z^{\epsilon}:=u_{x x}^{\epsilon}$ it follows that

$$
\left\{\begin{array}{l}
z_{t}^{\epsilon}=B z_{x x}^{\epsilon}+F^{\epsilon}, \\
z^{\epsilon}=0 \text { if } x \in\{0,1\},
\end{array}\right.
$$

where $F^{\epsilon}:=w_{t}^{\epsilon}$ or $F^{\epsilon}:=w_{x x}^{\epsilon}$. In both cases, by (3.8) and (3.13)

$$
\left\|z^{\epsilon}(0)\right\|_{H^{1}(0.1)} \leq C, \quad \int_{0}^{T}\left\|F^{\epsilon}(t)\right\|_{L^{2}(0.1)}^{2} d t \leq C,
$$

and so, applying (3.12) to each one of the sequences $\left\{z^{\epsilon}\right\}$ defined above we obtain (3.10). 
4. Numerical simulation. In this section we use a finite difference scheme to illustrate the behavior of solutions of (2.1), (2.2) with initial conditions

$$
\begin{cases}u^{\epsilon}(x, 0)=0 & 0<x<1 \\ u_{t}^{\epsilon}(x, 0)=\sin ^{6}\left(\frac{1}{\epsilon} \pi x\right), & 0<x<1\end{cases}
$$

where $\epsilon$ is taken to be $1 / m$ with $m$ an integer. By Theorem 3.7 we know that $\left\{u_{x x}^{\epsilon}\right\}$ converges strongly in $L_{\text {loc }}^{2}\left(\mathcal{K}_{\infty}\right)$; hence we ask whether $\left\{u_{x x}^{\epsilon}\right\}$ converges strongly in $L^{2}\left(\mathcal{K}_{T}\right)$. The simulations carried out in Figs. 1 through 4 seem to indicate that this is true, in spite of the fact that a residual, bounded, boundary layer remains.

We also consider the sequence $\left\{u_{x t}^{\epsilon}\right\}$. Note that the stronger assumptions on the initial conditions made in Theorem 3.10 do not hold here. However, Figs. 5 through 9 lead us to believe that $\left\{u_{x t}^{\epsilon}\right\}$ converges strongly under the present weaker conditions.

First we describe briefly the difference scheme used. Taking $\Delta x:=\frac{1}{K-1}$ with $K$ an integer, we set $x_{k}:=(k-1 / 2) \Delta x, t^{n}:=n \Delta t$, and denote

$$
u_{k}^{n} \approx u^{\epsilon}\left(x_{k}, t^{n}\right)
$$

(suppressing the dependence on $\epsilon$ ). For the boundary conditions we require

$$
u_{0}^{n}=u_{1}^{n}=u_{K}^{n}=u_{K-1}^{n}=0,
$$

and for the initial conditions

$$
\left\{\begin{array}{l}
u_{k}^{0}=0 \\
u_{k}^{1}=\Delta t \sin ^{6}\left(\frac{1}{\epsilon} \pi x_{k}\right)
\end{array}\right.
$$

Then the difference scheme is

$$
\begin{aligned}
(\Delta t)^{-2} & \left(u_{k}^{n+1}-2 u_{k}^{n}+u_{k}^{n-1}\right) \\
= & \beta(\Delta t)^{-1}\left[\frac{u_{k+1}^{n+1}-2 u_{k}^{n+1}+u_{k-1}^{n+1}}{(\Delta x)^{2}}-\frac{u_{k+1}^{n}-2 u_{k}^{n}+u_{k-1}^{n}}{(\Delta x)^{2}}\right] \\
& -\gamma \frac{u_{k+2}^{n+1}-4 u_{k+1}^{n+1}+6 u_{k}^{n+1}-4 u_{k-1}^{n+1}+u_{k-2}^{n+1}}{(\Delta x)^{4}} \\
& -\alpha u_{k}^{n}+\frac{\left[\left(D_{k+1 / 2}^{n}\right)^{3}-D_{k+1 / 2}^{n}\right]-\left[\left(D_{k-1 / 2}^{n}\right)^{3}-D_{k-1 / 2}^{n}\right]}{\Delta x}
\end{aligned}
$$

where

$$
D_{k+1 / 2}^{n}:=\frac{u_{k+1}^{n}-u_{k}^{n}}{\Delta x} .
$$

This scheme is second order in space but only first order in time. The time stepping method was chosen because it is unconditionally stable and its Fourier analysis (on the whole line with only the principle part of the equation) closely resembles that of the continuous equation; that is, high frequency modes decay rapidly in both.

Now we consider the solution to (2.1) with $\beta=3$ and $\gamma=\alpha=1$. Figures 1 through 4 show the graphs of

$$
x \mapsto 10^{3} u_{x x}^{\epsilon}\left(x, 5 \times 10^{-5}\right)
$$




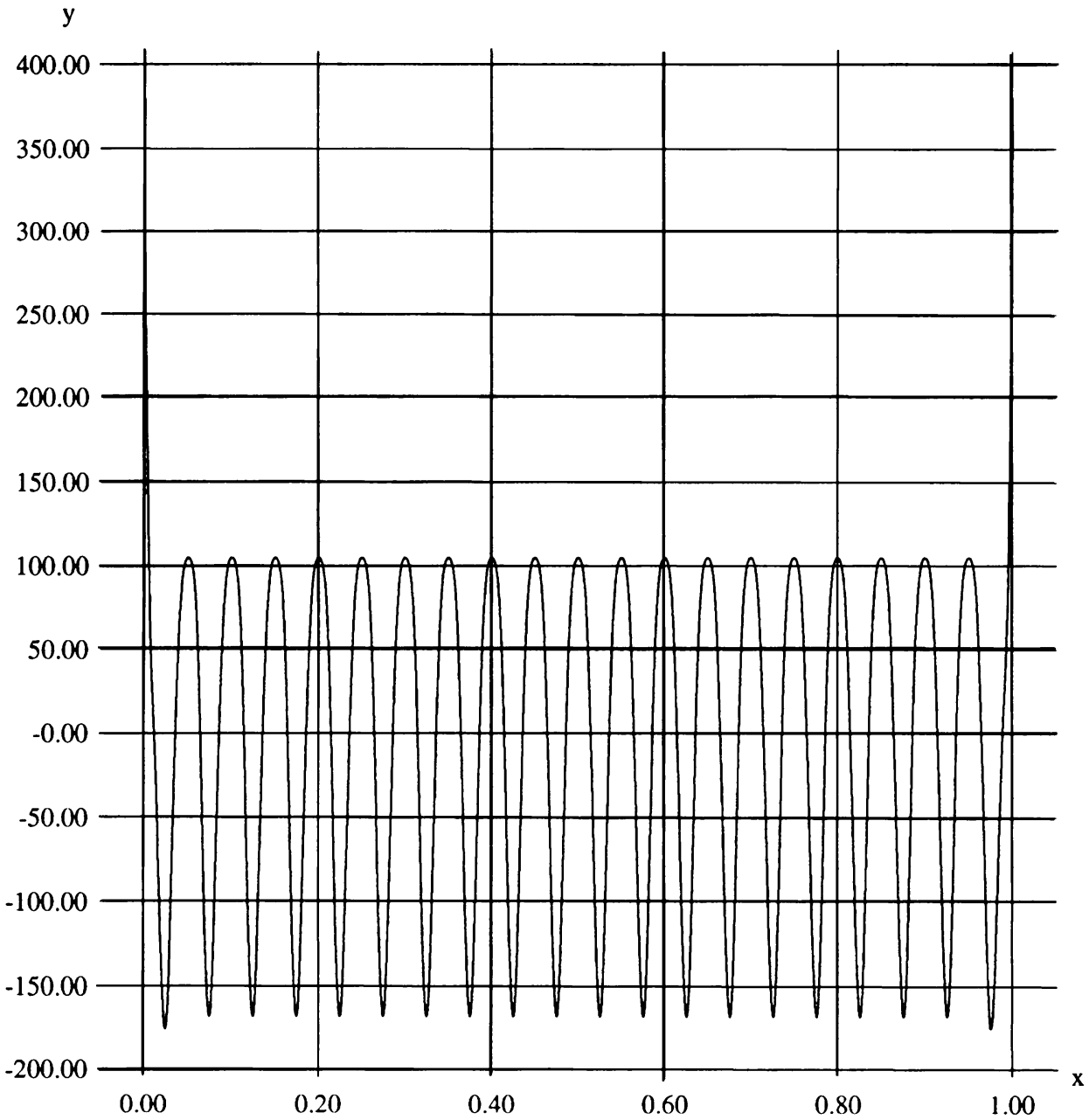

FIG. 1. $u(x, 0)=0, u_{t}(x, 0)=\sin ^{6}(20 \pi x), \beta=3, \gamma=\alpha=1$, $\Delta t=1.25 \times 10^{-6}, \Delta x=1 / 4000, y \times 10^{-3}=u_{x x}\left(x, 5 \times 10^{-5}\right)$.

for $\epsilon=1 / 20,1 / 40,1 / 80,1 / 160$, respectively. It appears that $\left\{u_{x: x}^{\mathrm{i}}\right\}$ does converge strongly in $L^{2}\left(\mathcal{K}_{T}\right)$, but that the limit (as $\epsilon \rightarrow 0$ ) has more interesting behavior near the boundary. Figures 5 through 9 display the graphs of

$$
x \mapsto u_{x t}^{c}\left(x, 10^{-5}\right)
$$

for $\epsilon=1 / 20,1 / 40,1 / 80,1 / 160,1 / 320$, respectively. These suggest that for fixed $t>0$

$$
x \mapsto u_{x t}^{\epsilon}(x, t)
$$

converges strongly in $L^{\propto}(0,1)$. Note that

$$
x \mapsto u_{x t}^{\epsilon}(x, 0)
$$

is unbounded in $L^{\propto}(0,1)$. 


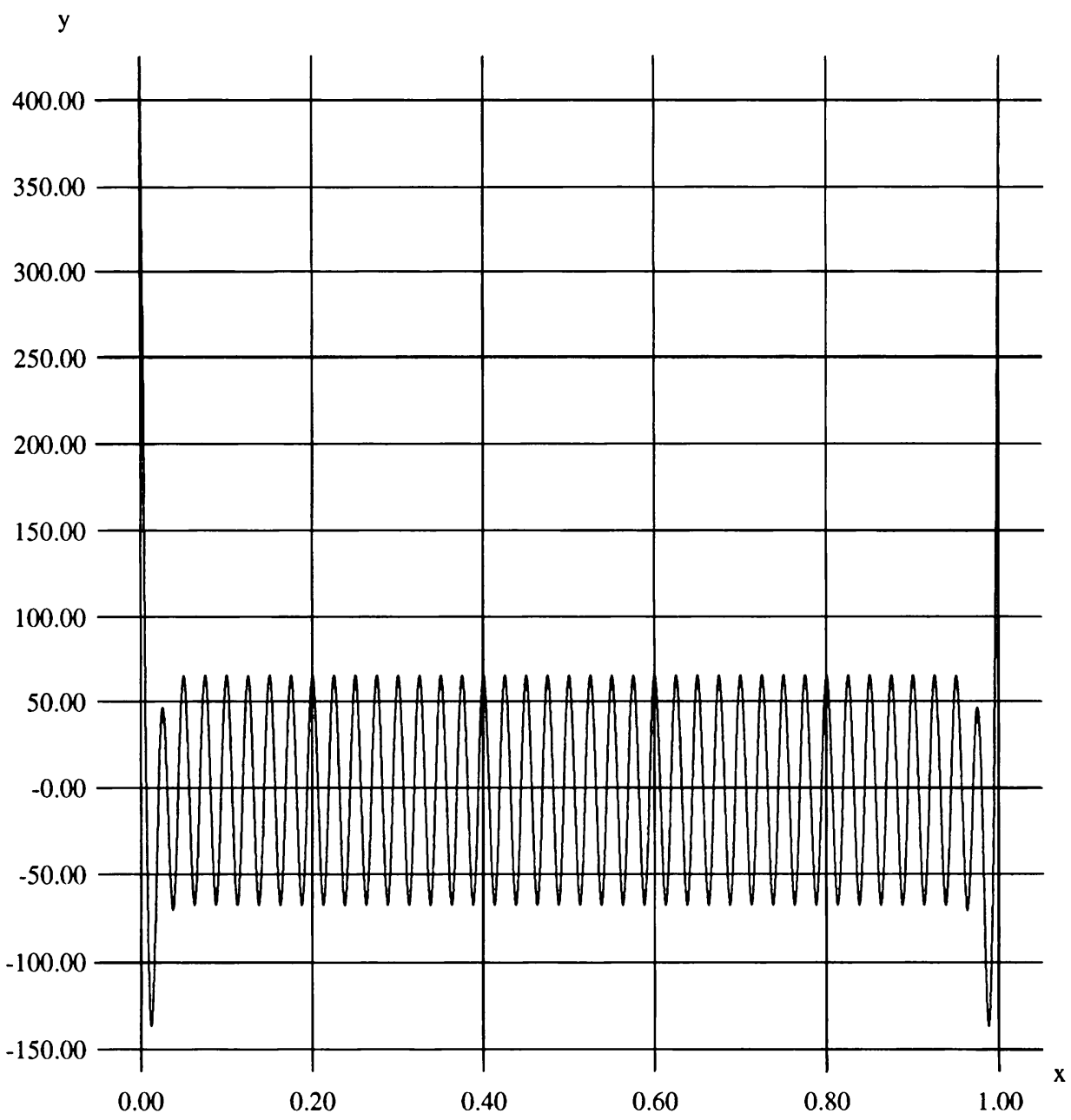

FIG. 2. $u(x, 0)=0, u_{t}(x, 0)=\sin ^{6}(40 \pi x), \beta=3, \gamma=\alpha=1$, $\Delta t=3.125 \times 10^{-7}, \Delta x=1 / 8000, y \times 10^{-3}=u_{x x}\left(x, 5 \times 10^{-5}\right)$. 


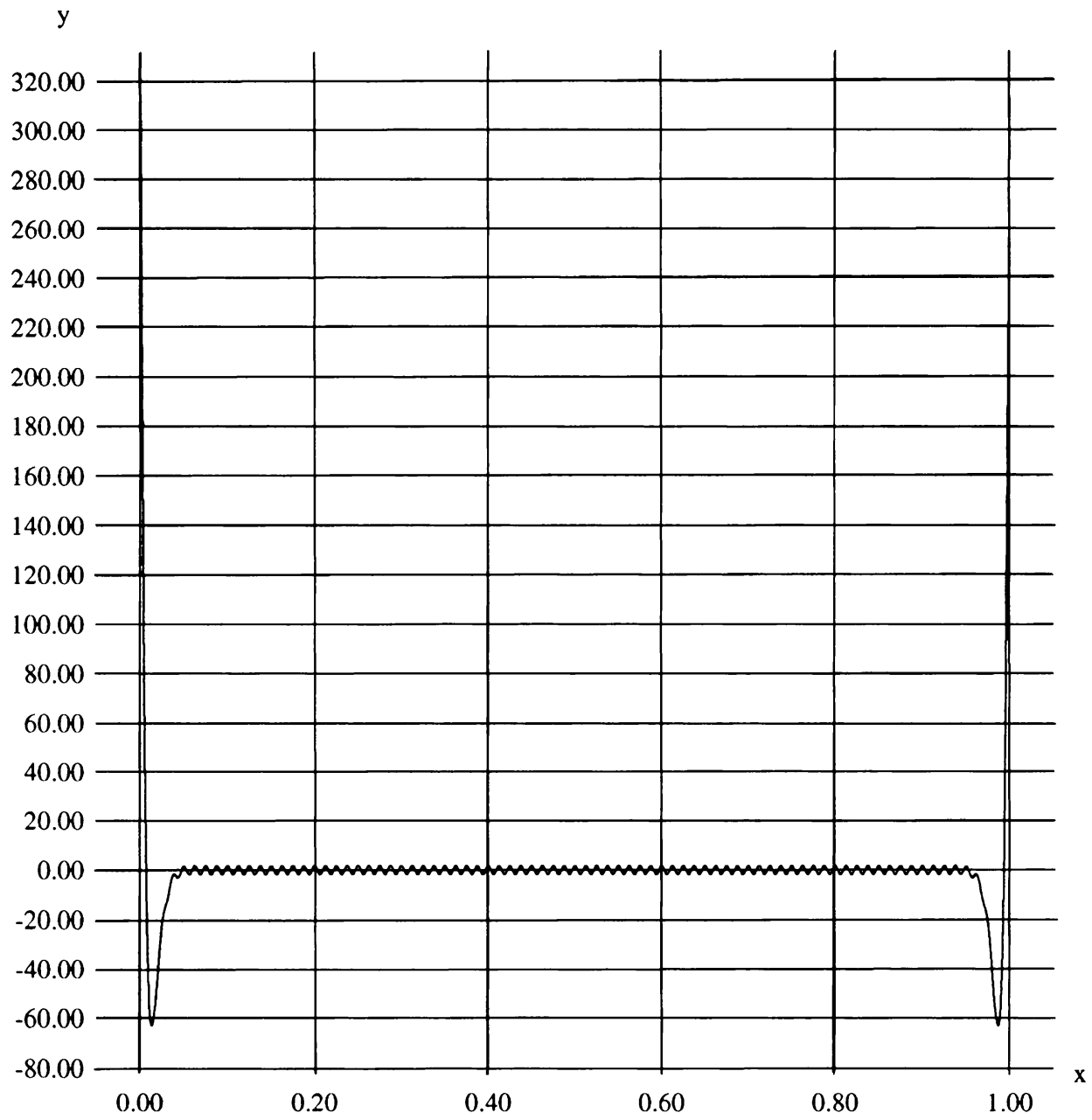

FIG. 3. $u(x, 0)=0, u_{t}(x, 0)=\sin ^{6}(80 \pi x), \beta=3, \gamma=\alpha=1$, $\Delta t=7.8125 \times 10^{-8}, \Delta x=1 / 16000, y \times 10^{-3}=u_{x x}\left(x, 5 \times 10^{-5}\right)$. 


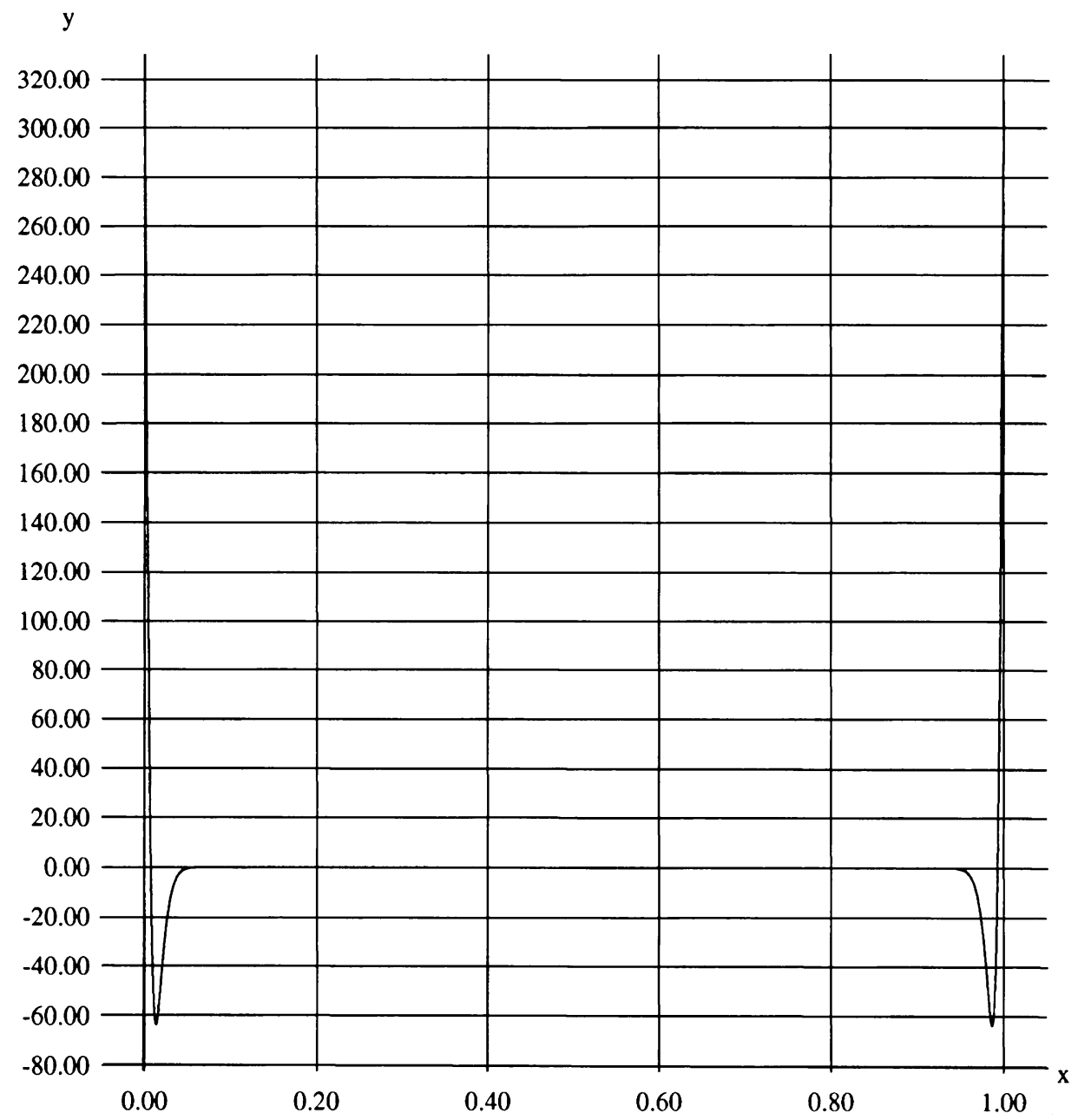

FIG. 4. $u(x, 0)=0, u_{t}(x, 0)=\sin ^{6}(160 \pi x), \beta=3, \gamma=\alpha=1$, $\Delta t=1.953125, \Delta x=1 / 32000, y \times 10^{-3}=u_{x x}\left(x, 5 \times 10^{-5}\right)$. 
y

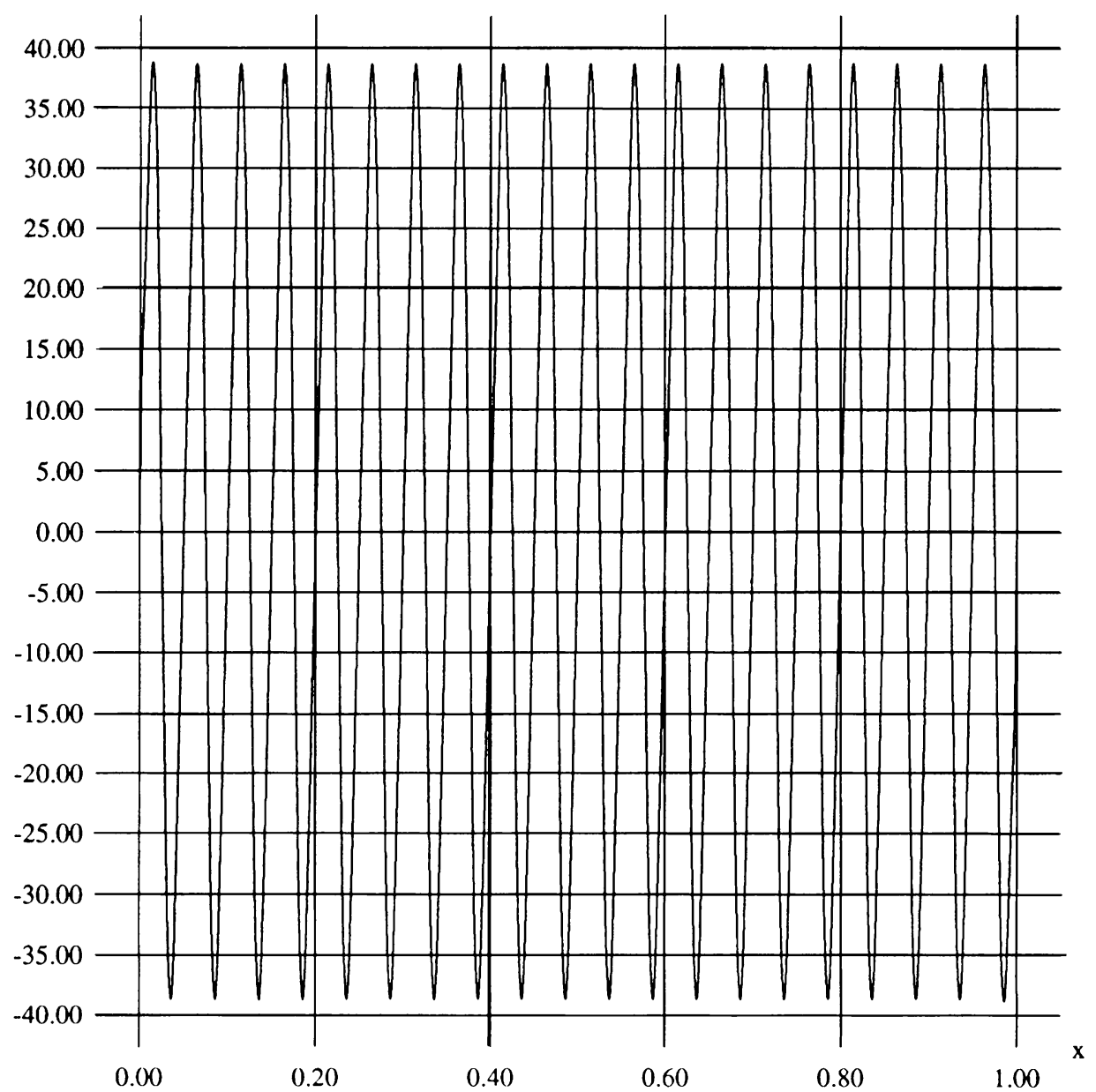

FIG. 5. $u(x, 0)=0, u_{t}(x, 0)=\sin ^{6}(20 \pi x), \beta=3, \gamma=\alpha=1$,

$\Delta t=3.125 \times 10^{-\tau}, \Delta x=1 / 8000, y=u_{x t}\left(x, 10^{-5}\right)$. 
y

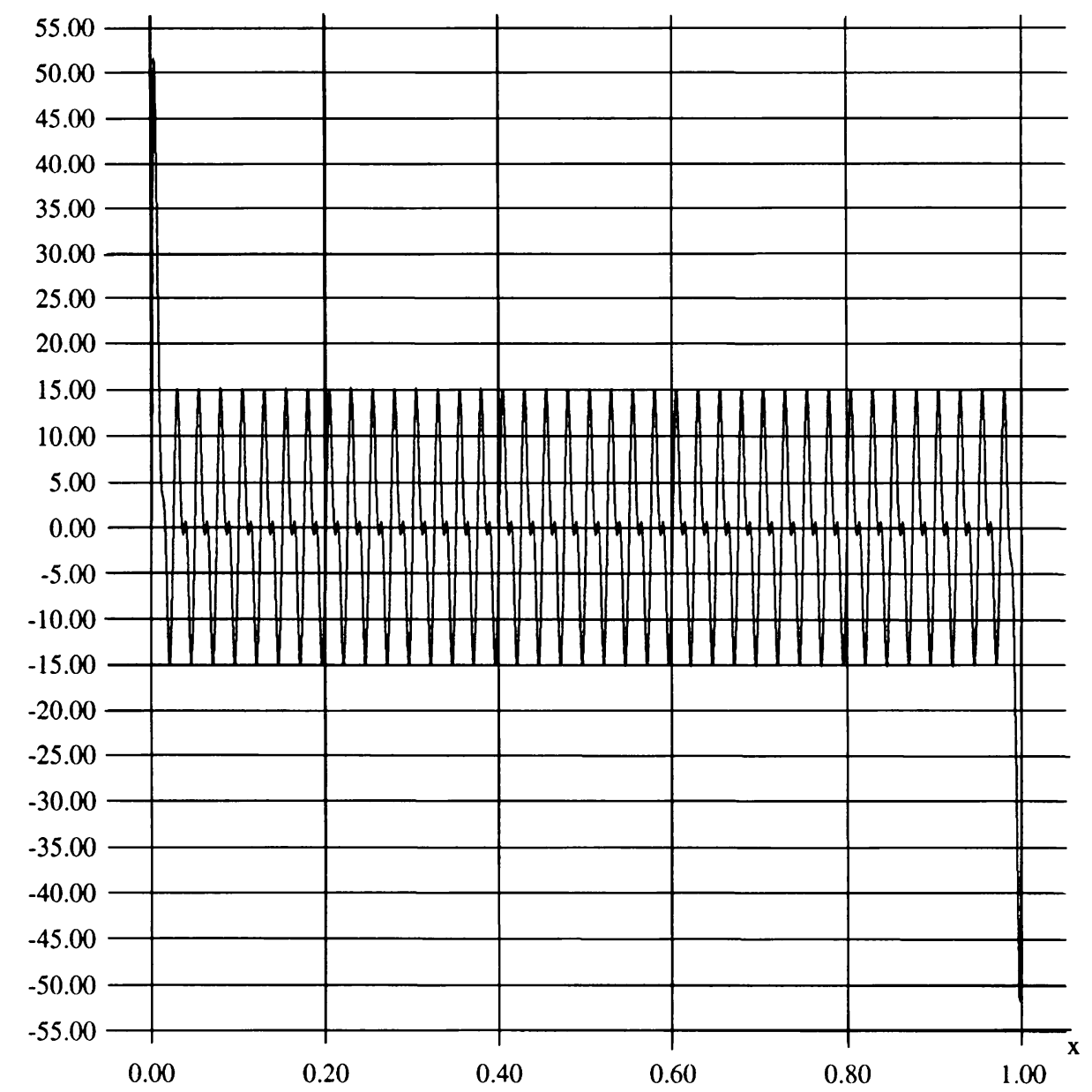

FIG. 6. $u(x, 0)=0, u_{t}(x, 0)=\sin ^{6}(40 \pi x), \beta=3, \gamma=\alpha=1$, $\Delta t=7.8125 \times 10^{-8}, \Delta x=1 / 16000, y=u_{x t}\left(x, 10^{-5}\right)$. 
y

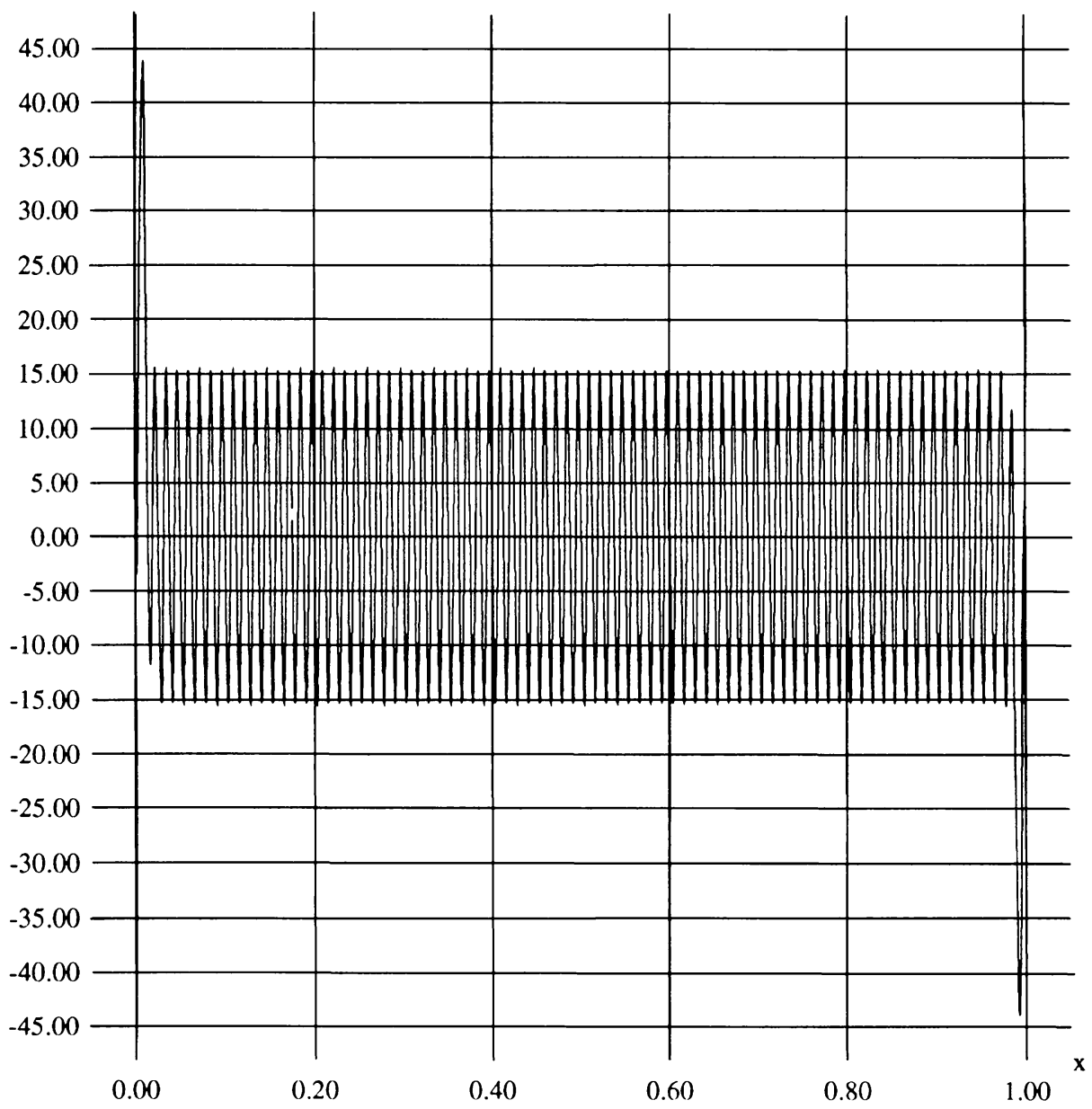

FIG. 7. $u(x, 0)=0, u_{t}(x, 0)=\sin ^{6}(80 \pi x), \beta=3, \gamma=\alpha=1$, $\Delta t=1.953125 \times 10^{-8}, \Delta x=1 / 32000, y=u_{x t}\left(x, 10^{-5}\right)$. 
y

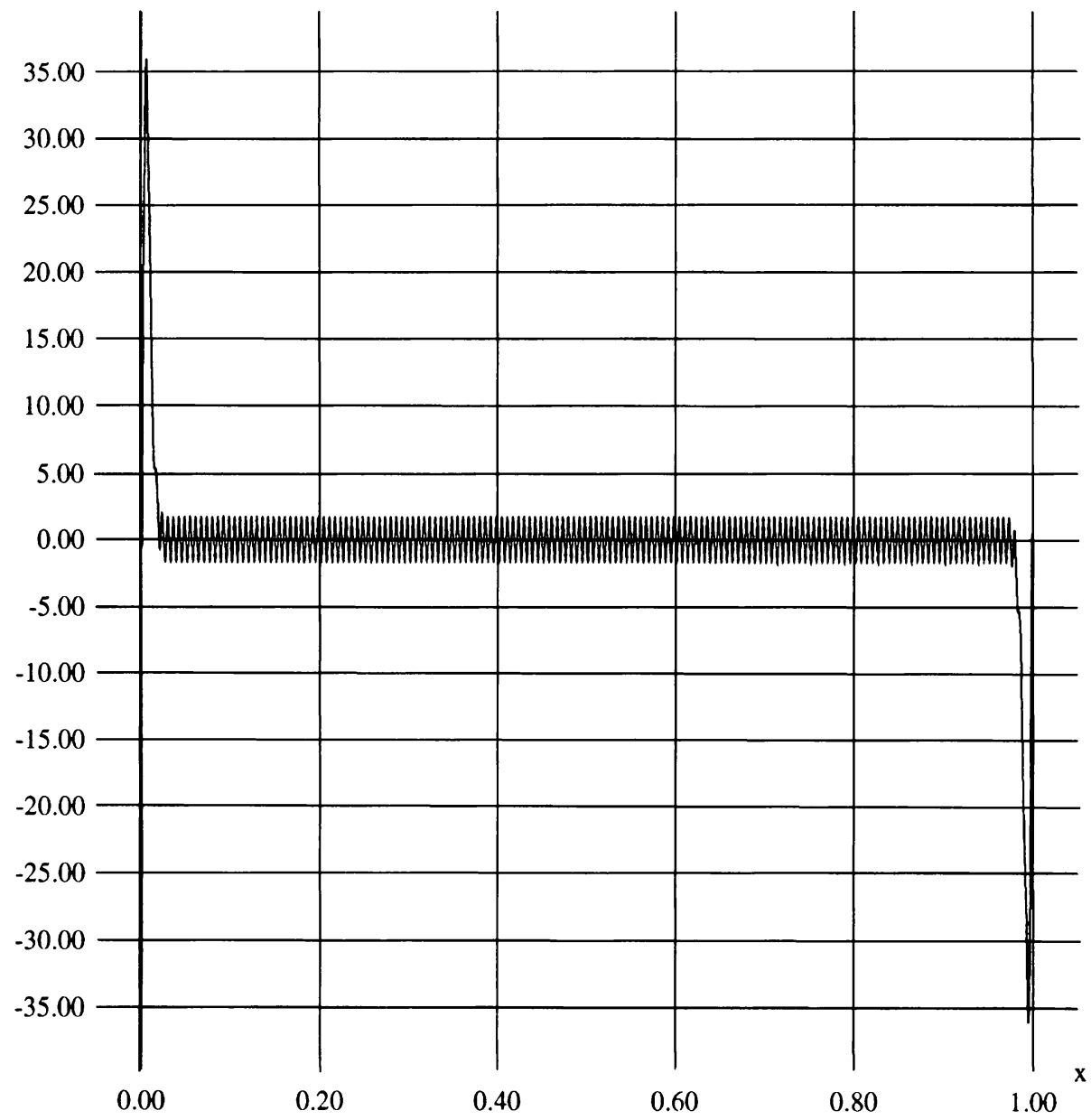

FIG. 8. $u(x, 0)=0, u_{t}(x, 0)=\sin ^{6}(160 \pi x), \beta=3, \gamma=\alpha=1$, $\Delta t=4.8828125 \times 10^{-9}, \Delta x=1 / 64000, y=u_{x t}\left(x, 10^{-5}\right)$. 
y

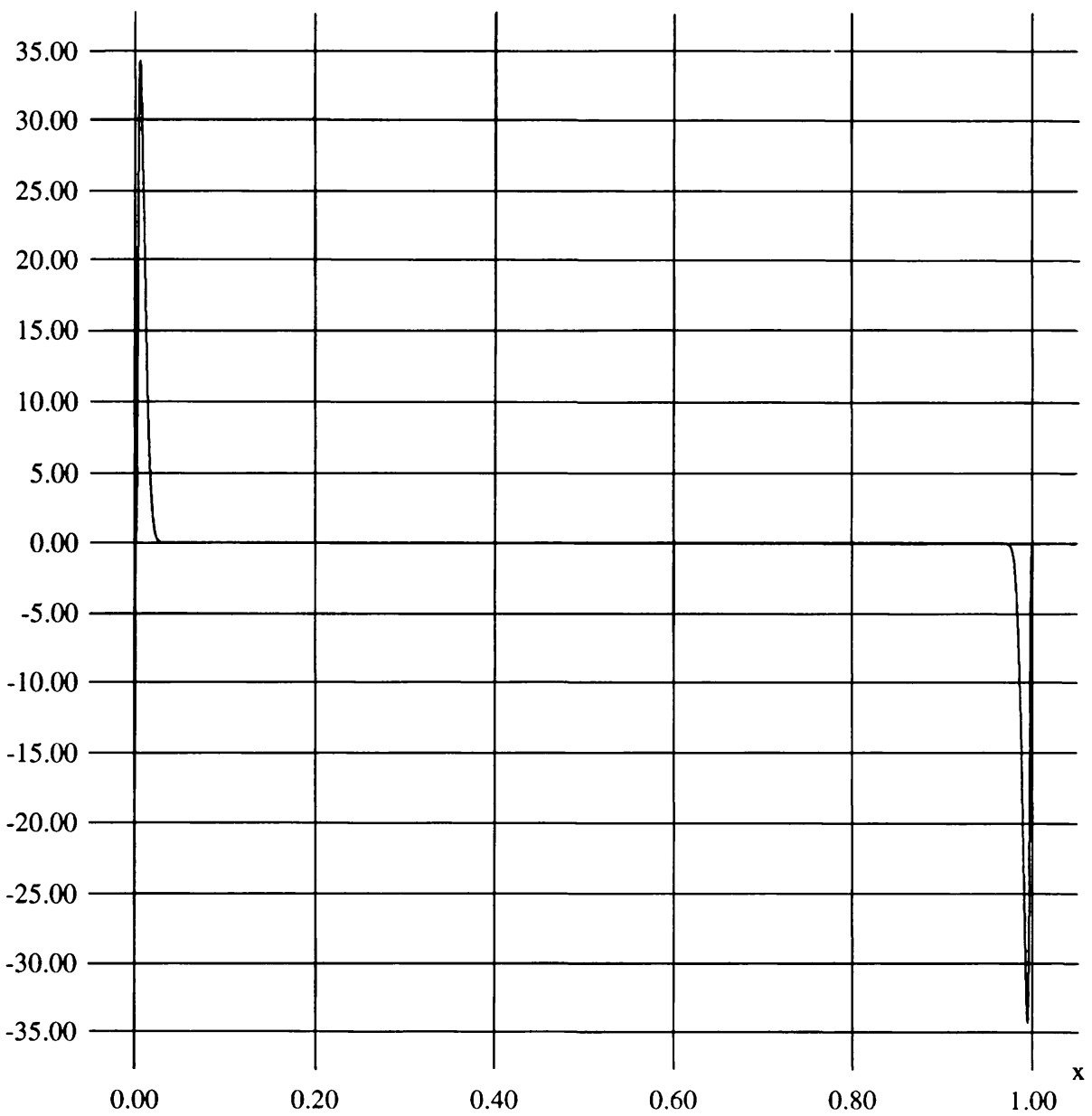

FIG. 9. $u(x, 0)=0, u_{t}(x, 0)=\sin ^{6}(320 \pi x), \beta=3, \gamma=\alpha=1$, $\Delta t=1.220703125 \times 10^{-9}, \Delta x=1 / 128000, y=u_{x t}\left(x, 10^{-5}\right)$.

Acknowledgments. The research of I. Fonseca and M. Shvartsman was partially supported by the Army Research Office and the National Science Foundation through the Center for Nonlinear Analysis. In addition, the research of I. Fonseca was also partially supported by the National Science Foundation under Grant No. DMS-9500531. The authors are indebted to L. Tartar and W. Hrusa for many fruitful and stimulating discussions on the subject of this work. 


\section{REFERENCES}

[A] J. P. Aubin, Un théorème de compacité, C. R. Acad. Sci. 256, 5042-5044 (1963)

[BHJPS] J. M. Ball, P. J. Holmes, R. D. James, R. L. Pego, and P. J. Swart, On the dynamics of fine structure, J. Nonlinear Science 1, 17-70 (1991)

[BFS] D. Brandon, I. Fonseca, and P. J. Swart, The creation and propagation of oscillatory microstructure in a dynamical model of displacive phase transformations, in Progress in Partial Differential Equations: the Metz Surveys 1994, 3, Pitman Res. Notes Math. Ser. 314, Longman Sci. Tech., Harlow, 1994, pp. 130-144

[E] L. C. Evans, Partial Differential Equations, Berkeley Mathematics Lecture Notes, 1994

[L] J. L. Lions, Quelques Méthodes de Résolution de Problèmes aux Limites non Linéaires, Dunod Gauthier-Villars, 1969

[M] F. Murat, A survey on compensated compactness, in Contributions to Modern Calculus of Variations, (ed. L. Cesari), Pitman Research Notes in Math., Longman, Harlow, 1987, pp. 145-183

[P] A. Pazy, Semigroups of linear operators and applications to partial differential equations, Springer-Verlag, New York, 1983

[Pe] R. L. Pego, Phase transitions in one-dimensional nonlinear viscoelasticity: Admissibility and stability, Arch. Rational Mech. Anal. 97, 353-394 (1987)

[T1] L. Tartar, The compensated compactness and applications to PDEs, in Nonlinear Analysis and Mechanics (ed. R. Knops), Pitman Research Notes in Math., Vol. 4, Pitman, London-San Francisco, Longman, Harlow, 1979, pp. 136-212

[T2] L. Tartar, Nonlinear partial differential equations using compactness method, Report 1584, MRC, Univ. Wisconsin, 1975.

[TZ] L. Truskinovsky and G. Zanzotto, Ericksen's bar revisited, Preprint, 1994 